Virginia Commonwealth University VCU Scholars Compass

2008

\title{
Correlation of tellurium inclusions and carrier lifetime in detector grade cadmium zinc telluride
}

Ezzat S. Elshazly

Virginia Commonwealth University

Gary C. Tepper

Virginia Commonwealth University, gctepper@vcu.edu

Follow this and additional works at: http://scholarscompass.vcu.edu/egmn_pubs

Part of the Mechanical Engineering Commons, and the Nuclear Engineering Commons

Elshazly, E. and Tepper, G.C. Correlation of tellurium inclusions and carrier lifetime in detector grade cadmium zinc telluride. Applied Physics Letters, 93, 042112 (2008). Copyright (C) 2008 AIP Publishing LLC.

\section{Downloaded from}

http://scholarscompass.vcu.edu/egmn_pubs/19

This Article is brought to you for free and open access by the Dept. of Mechanical and Nuclear Engineering at VCU Scholars Compass. It has been accepted for inclusion in Mechanical and Nuclear Engineering Publications by an authorized administrator of VCU Scholars Compass. For more information, please contact libcompass@vcu.edu. 


\title{
Correlation of tellurium inclusions and carrier lifetime in detector grade cadmium zinc telluride
}

\author{
Ezzat S. Elshazly and Gary Tepper ${ }^{a)}$ \\ Department of Mechanical Engineering, Virginia Commonwealth University, Richmond, Virginia 23284, \\ USA
}

(Received 8 April 2008; accepted 15 July 2008; published online 31 July 2008)

\begin{abstract}
Carrier lifetimes and tellurium inclusion densities in detector grade cadmium zinc telluride crystals grown by the high pressure Bridgman method were optically measured using pulsed laser microwave cavity perturbation and infrared microscopy. Excess carriers were produced in the material using a pulsed laser with a wavelength of $1064 \mathrm{~nm}$ and pulse width of $7 \mathrm{ns,} \mathrm{and} \mathrm{the}$ electronic decay was measured at room temperature. Spatial mapping of lifetimes and defect densities in cadmium zinc telluride was performed to determine the relationship between tellurium defect density and trapping. A strong correlation was found between the volume fraction of tellurium inclusions and the carrier trapping time. (C) 2008 American Institute of Physics.
\end{abstract}

[DOI: $10.1063 / 1.2967726]$

Cadmium zinc telluride (CZT) $\left(\mathrm{Cd}_{1-x} \mathrm{Zn}_{x} \mathrm{Te}, x \sim 0.1\right)$ is an important semiconductor material for use as a room temperature radiation detector. ${ }^{1-4}$ The material density $\left(5.78 \mathrm{~g} / \mathrm{cm}^{3}\right)$ and average atomic number (49.1) are sufficient to provide reasonably high gamma-ray stopping power. The bandgap is wide enough $(1.6 \mathrm{eV})$ to suppress thermal generation of charge carriers at room temperature and under proper growth conditions the material quality can be sufficient to provide high resistivity $\left(10^{10} \Omega \mathrm{cm}\right)$ and low leakage currents under applied fields in the $10^{3} \mathrm{~V} / \mathrm{cm}$ range. The mean free path of the charge carriers, which establishes the maximum detector thickness, is given by the product of the charge carrier mobility, trapping time, and applied electric field, or $\mu \tau E$. The mean free path of electrons is currently in the range of a few centimeters, and there is a significant effort underway to increase the volume and spectroscopic performance of CZT detectors for applications in nuclear nonproliferation and homeland security.

It is well established that crystallographic defects such as grain boundaries, dislocations, and tellurium inclusions can trap charge and degrade the spectroscopic performance of CZT gamma-ray spectrometers. ${ }^{5,6}$ Of the various defects present in CZT, tellurium inclusions have been shown to have a particularly detrimental impact on charge collection and spectroscopic performance. ${ }^{6-13}$ Detectors fabricated from CZT with a large number of Te inclusions exhibited lower energy resolution in comparison with detectors fabricated from higher quality material. In addition, high resolution $\mathrm{x}$-ray mapping studies have recently shown that charge collection is specifically impeded along crystallographic trajectories containing tellurium inclusions. ${ }^{3,6}$ However, charge collection studies require sample metallization and do not provide a direct measurement of the carrier lifetime, making it challenging to directly relate the tellurium inclusions to the carrier trapping time.

In this paper, we report on a direct correlation between the volume fraction of the tellurium inclusions and the carrier trapping time in detector grade $\mathrm{Cd}_{1-x} \mathrm{Zn}_{x} \mathrm{Te}(x \sim 0.1)$. The

${ }^{a)}$ Electronic mail: gctepper@vcu.edu. crystals were grown by the high pressure Bridgman method at $\mathrm{eV}$ Products and have dimensions of $10 \times 10 \times 10 \mathrm{~mm}^{3}$. The size and number density of the tellurium inclusions were measured using infrared (IR) microscopy. The trapping times were measured using a pulsed laser microwave cavity perturbation method described in detail in a previous publication. ${ }^{14}$

To measure the trapping time, the cubic CZT crystals were mounted on a ceramic $\mathrm{BeO}$ rod and inserted axially into the center of a cylindrical $\mathrm{TE}_{011}$ microwave resonant cavity with a resonant frequency near $5 \mathrm{GHz}$ and a maximum power of $100 \mathrm{~mW}$. The loaded cavity was critically coupled, and the reflected microwave power was monitored as a function of time during pulsed laser excitation of the sample. A pulsed neodymium-doped yttrium aluminum garnet laser with wavelength of $1064 \mathrm{~nm}$ and a pulse width of 7 ns was used to excite carriers in the CZT crystal through a 1 $\mathrm{mm}$ diameter aperture in the cavity wall. The excitation wavelength of the laser is below the bandgap energy of the CZT so that carrier generation is from midgap energy states within the bulk of the crystal.

An IR microscope imaging system was used for measuring the Te inclusions in the CZT crystals. The CZT is trans-

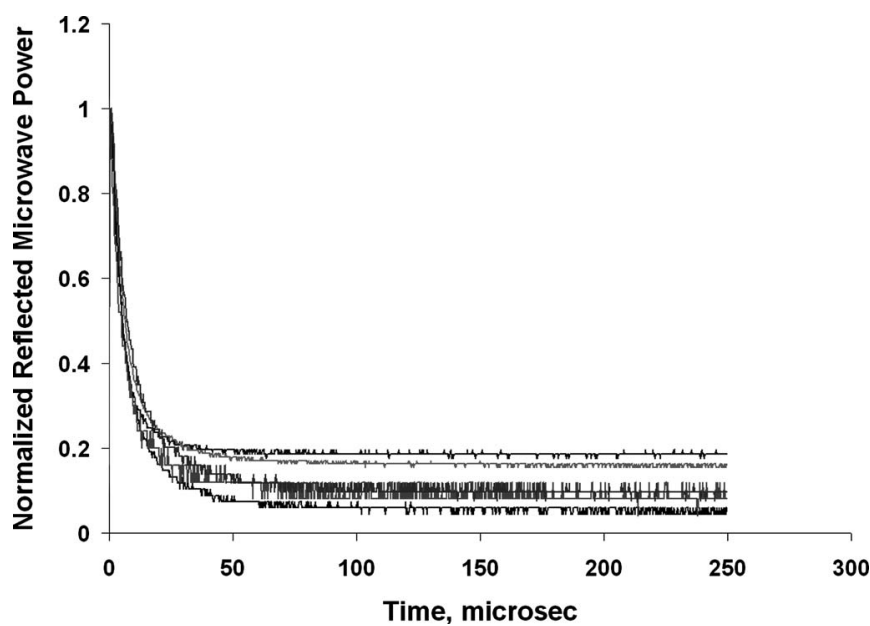

FIG. 1. Normalized reflected microwave power vs time for CZT1 at room temperature. 


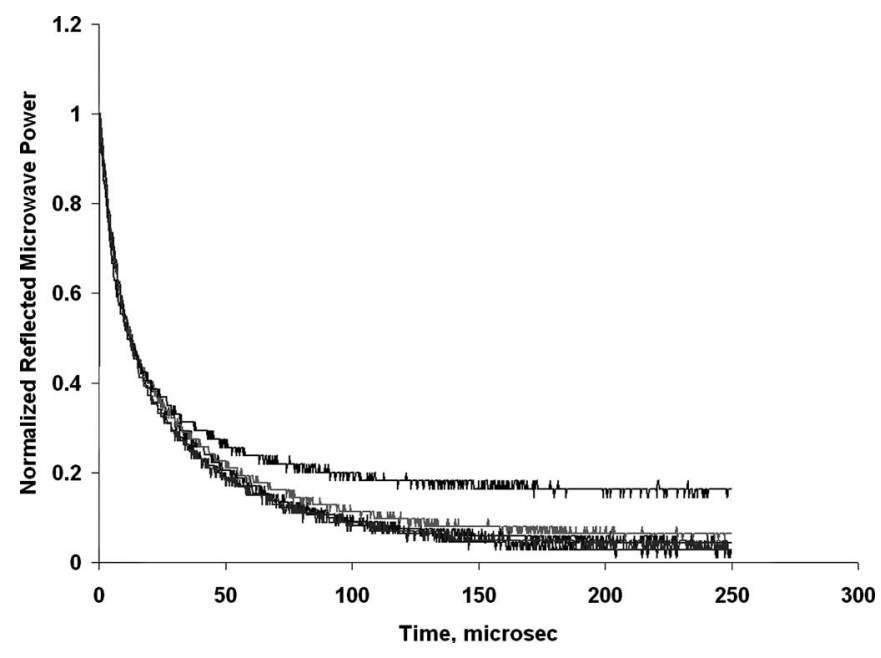

FIG. 2. Normalized reflected microwave power vs time for CZT2 at room temperature.

parent to IR light, whereas the tellurium inclusions are opaque. The system consists of an IR back light, threedimensional (3D) translation stage, and IR sensitive digital camera with long objective microscope lens and a 7.8 $\times 10.6 \mathrm{~mm}^{2}$ sensor with $2208 \times 3000$ pixels. The $3 \mathrm{D}$ translation stage was used to change the $X Y Z$ position of the $\mathrm{CZT}$ crystal with respect to the camera and images were collected and compiled from planes parallel to the cube surfaces. IR images were saved as monochrome high resolution jpeg files, and IMAGE-PRO PLUS software version 6 was used to calculate the size and the concentration of Te precipitates in the crystal.

Figures 1 and 2 show a set of decay curves (normalized reflected microwave power versus time) obtained after pulsed excitation of the two CZT samples at random locations. The reflected microwave pulse is a measurement of the free charge carrier density as a function of time after laser excitation, and the carrier trapping time was determined by fitting the decay curves to an exponential function with an adjustable time constant. The laser probes a cylindrical volume of the sample, $1 \mathrm{~mm}$ in diameter and $10 \mathrm{~mm}$ long and is, therefore, a measurement of the cumulative effect of charge traps within the measurement volume. The first sample,

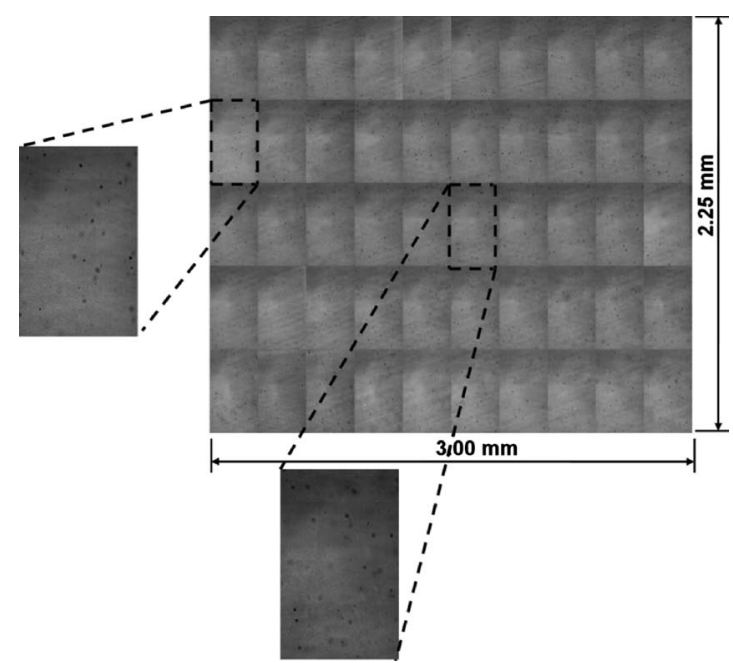

FIG. 4. IR microscope images of CZT2 at $10 \times$ magnification.

CZT1, had an average decay time of $5.5 \mu$ s and the second sample, CZT2, had an average decay time of $16.9 \mu$ s. For each sample, the decay time did not vary significantly throughout the volume of the crystal and is, therefore, considered to be representative.

Figures 3 and 4 show composite IR images obtained at $10 \times$ magnification from typical $3 \times 2.25 \mathrm{~mm}^{2}$ planes within CZT1 and CZT2, respectively. Because CZT is transparent to IR light and tellurium is not, the tellurium inclusions show up as distinct black spots under the IR back lighting and were found to be distributed uniformly throughout the bulk of the crystal. Figure 5 is a histogram of the size distribution of tellurium inclusions compiled from two arbitrary planes in each sample.

The tellurium planar defect density in CZT1 is 89 inclusions $/ \mathrm{mm}^{2}$ with an average inclusion diameter of $27 \mu \mathrm{m}$. CZT2 has a planar defect density of 108 inclusions $/ \mathrm{mm}^{2}$ with an average inclusion diameter of $18 \mu \mathrm{m}$. The volume fraction of the tellurium inclusions (fractional crystal volume occupied) was estimated assuming that the defects are spherical and was $3.4 \%$ and $1.8 \%$ for CZT1 and CZT2, respectively.

From the imaging and lifetime data of Figs. 1-5, there appears to be a correlation between the volume fraction of
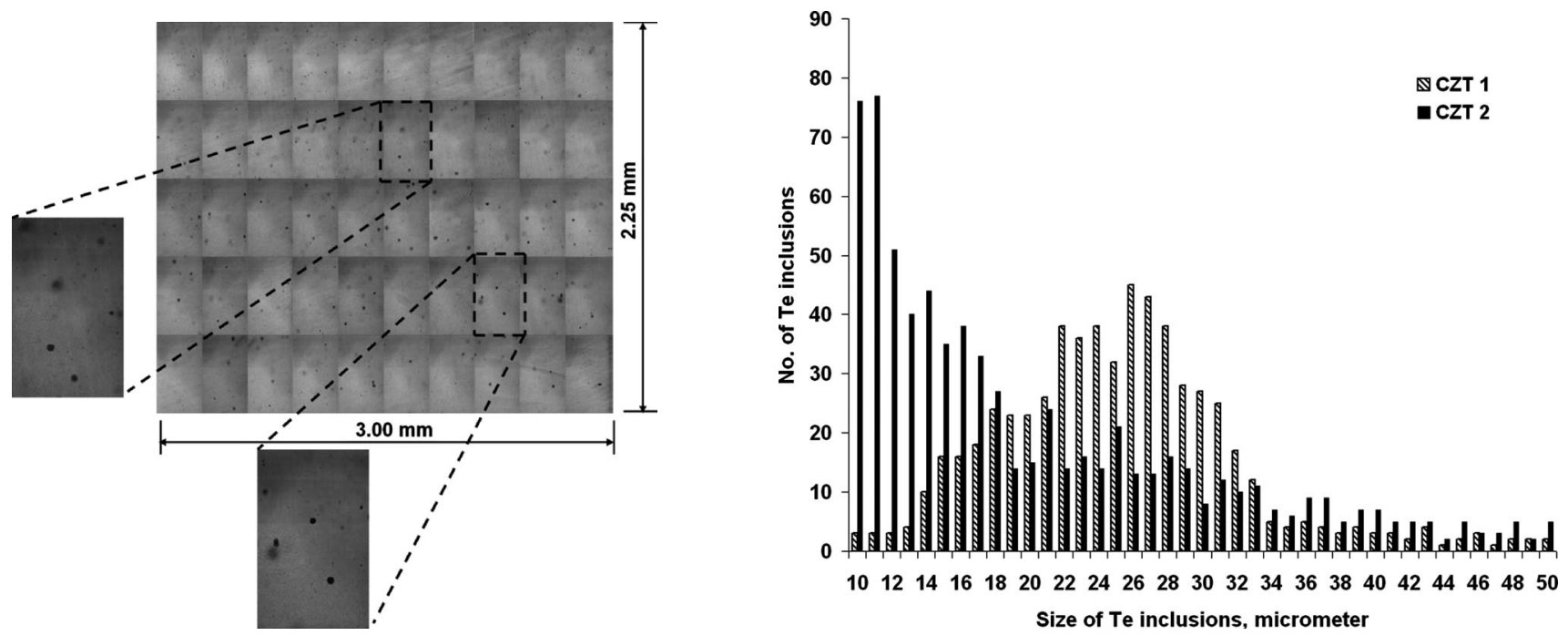
the tellurium inclusions and the carrier trapping time in CZT. Sample CZT1 has a volume averaged trapping time of $5.5 \mu \mathrm{s}$ and a tellurium inclusion volume fraction of $3.4 \%$. On the other hand, sample CZT2 has a volume averaged trapping time of $16.9 \mu \mathrm{s}$ and a tellurium inclusion volume fraction of $1.8 \%$. That is, the carrier trapping time is significantly longer in the sample with a lower volume fraction of tellurium inclusions.

While it is tempting to conclude that this correlation is a direct result of the Te inclusions acting as highly efficient trapping sites, there are other possible explanations for this correlation that cannot be ruled out at this time. The Te inclusions are intensely studied, in part because they are so readily visible in CZT. However, Te inclusions and precipitates are strongly associated with the presence of many other kinds of defects and charge traps in CZT, and the presence of large diameter Te inclusions may simply be a measure of the overall crystal quality and it is likely that other unseen defects are contributing to charge trapping.

Both the lifetime and defect measurements reported here were performed using simple, contactless optical methods and do not require any specific sample preparation or metallization. We, therefore, believe that these lifetime/defect density measurements can be used as a rapid material characterization/screening tool to select materials for specific detector applications.
Funding of this work was provided by the Department of Homeland Security, Domestic Nuclear Detection Office.

${ }^{1}$ T. E. Schlesinger, J. E. Toney, H. Yoon, E. Y. Lee, B. A. Brunett, L. Franks, and R. B. James, Mater. Sci. Eng., R. 32, 103 (2001).

${ }^{2}$ H. Chen, S. A. Awadalla, K. Iniewski, P. H. Lu, F. Harris, J. Mackenzie, T. Hasanen, W. Chen, R. Redden, G. Bindley, I. Kuvvetli, C. B. Jorgensen, P. Luke, M. Amman, J. S. Lee, A. E. Bolotnikov, G. S. Camarda, Y. Cui, A. Hossain, and R. B. James, J. Appl. Phys. 103, 014903 (2008).

${ }^{3}$ G. A. Carini, A. E. Bolotnikov, G. S. Camarda, and R. B. James, Nucl. Instrum. Methods Phys. Res. A 579, 120 (2007).

${ }^{4}$ R. Kessick, G. Tepper, E. Lee, and R. James, J. Appl. Phys. 87, 2408 (2000)

${ }^{5}$ G. A. Carini, A. E. Bolotnikov, G. S. Camarda, G. W. Wright, R. B. James, and L. Li, Appl. Phys. Lett. 88, 143515 (2006).

${ }^{6}$ P. N. Luke, M. Amman, and J. S. Lee, IEEE Trans. Nucl. Sci. 51, 1199 (2004).

${ }^{7}$ R. B. James, T. E. Schlesinger, L. C. Lund, and M. Schieber, Semiconductors for Room Temperature Nuclear Detector Applications (Academic New York, 1995), Vol. 43.

${ }^{8}$ P. Rudolph and M. Mühlberg, Mater. Sci. Eng., B 16, 8 (1993).

${ }^{9}$ M. C. Duff, D. B. Hunter, P. Nuessle, D. R. Black, H. Burdette, J. Woicik, A. Burger, and M. Groza, J. Electron. Mater. 36, 1092 (2007).

${ }^{10}$ A. E. Bolotnikov, G. S. Camarda, G. A. Carini, Y. Cui, L. Li, and R. B. James, Nucl. Instrum. Methods Phys. Res. A 571, 687 (2007).

${ }^{11}$ C. Szeles, S. E. Cameron, S. A. Soldner, J.-O. Ndap, and M. D. Reed, J Electron. Mater. 33, 742 (2004).

${ }^{12}$ C. Szeles, S. E. Cameron, J.-O. Ndap, and W. C. Chalmers, IEEE Trans. Nucl. Sci. 49, 2535 (2002).

${ }^{13}$ C. Szeles, D. Bale, J. Grosholz, G. L. Smith, M. Blostein, and J. Eger, Proc. SPIE 6319, 191 (2006).

${ }^{14}$ G. Tepper, R. Kessick, and C. Szeles, Proc. SPIE 4507, 79 (2001) 